\title{
A Simple Prediction Model of Hypoxemia During One-Lung Ventilation
}

Motoki Namekawa MD, Norihito Okumura² MD, Shigeki Yamashita ${ }^{1}$ MD

${ }^{1}$ Department of Anesthesiology, Kurashiki Central Hospital ${ }^{2}$ Department of Thoracic Surgery, Kurashiki Central Hospital

\section{【Background and Aim】}

- One-lung ventilation (OLV) may cause severe hypoxemia during thoracic surgery.

- No single factor independently accurately predicts hypoxemia during OLV.

- We tried to construct a simple prediction model of hypoxemia during OLV by combining several relevant factors.

\section{(Methods】}

This single center, retrospective, observational study was approved by the Institutional Ethics Board of Kurashiki Central Hospital.

\section{Patient Randomization}

Derivation group ( $75 \%$ of patients): to develop the most suitable model

Validation group ( $25 \%$ of patients): to evaluate the validity of the model

\section{Definition of hypoxemia during OLV (either of the following)}

- $\mathrm{SpO}_{2}<91 \%$ with $100 \%$ oxygen

- Apneic oxygen insufflation or continuous positive airway pressure was required to non-dependent lung

- Intermittent two lung ventilation was required

\section{Factors Evaluated}

Body mass index $(\mathrm{BMI})^{1)}$, Preoperative $\mathrm{PaO}_{2}$ value ${ }^{2)}$, Previous lung resection on the dependent lung344),

Side of operation ${ }^{2)}$, Forced expiratory volume in one second (FEV $1.0 \%$ prediction) ${ }^{2)}$

\section{【Results】}

Figure 1. Patient Enrollment

Patients who underwent lung resection surgery

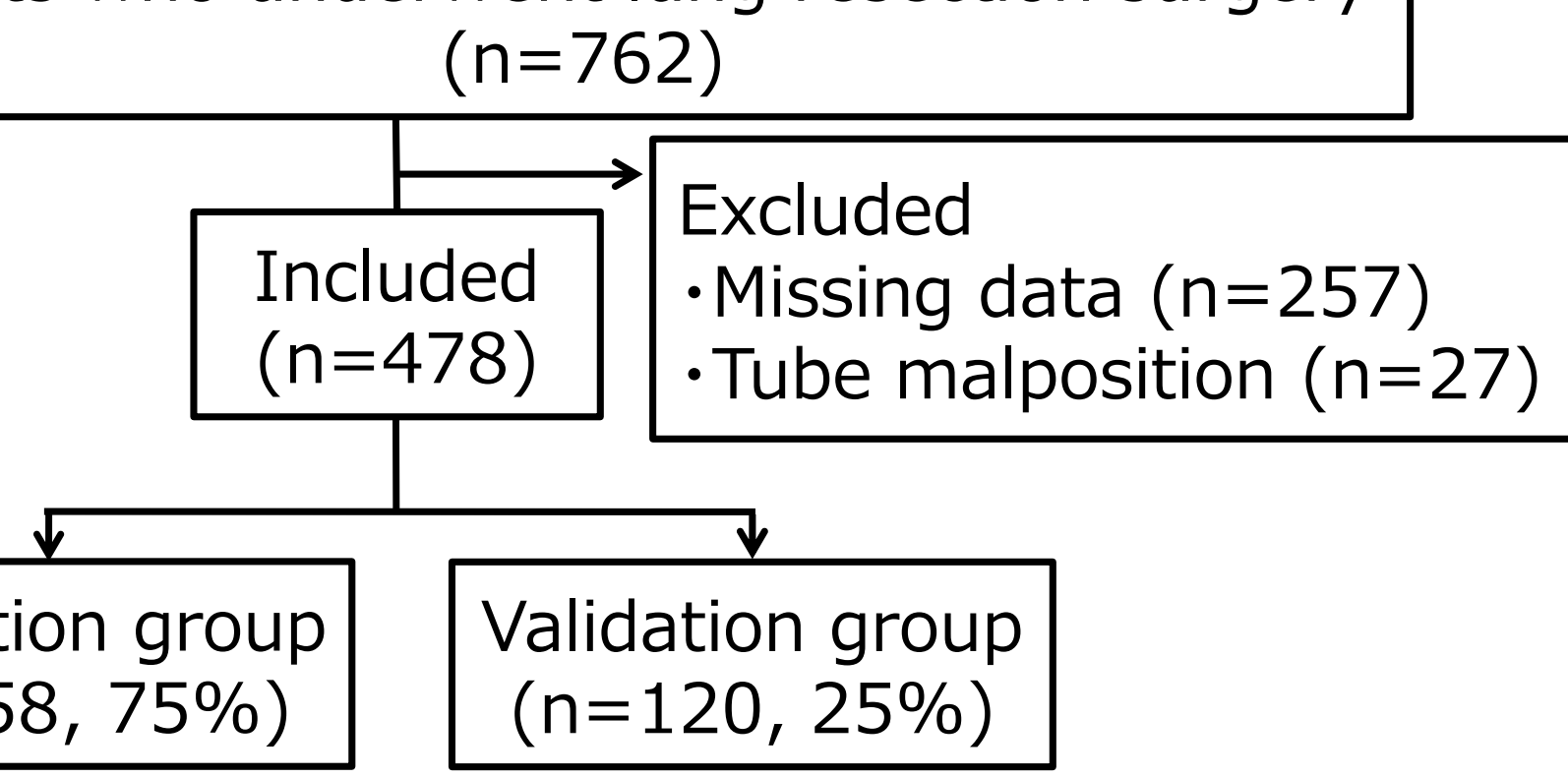

Table 1. Multivariable Model with All Factors

\begin{tabular}{|l|r|r|r|}
\hline & Odds ratio & \multicolumn{1}{c|}{$95 \%$ CI } & P value \\
\hline BMI & 1.07 & $0.949-1.20$ & 0.28 \\
\hline Low preoperative $\mathrm{PaO}_{2}$ & 0.939 & $0.906-0.974$ & $<0.001$ \\
\hline Previous lung resection & 13.6 & $3.94-46.7$ & $<0.001$ \\
\hline Right-side operation & 7.31 & $2.64-20.2$ & $<0.001$ \\
\hline FEV1.0 \%prediction & 0.99 & $0.959-1.02$ & 0.53
\end{tabular}

Table 2. Prediction Score* for Hypoxemia

\begin{tabular}{|c|r|l|l|}
\hline & Point & $\boldsymbol{\beta}$ coefficients & P value \\
\hline $\begin{array}{c}\text { Preoperative } \mathrm{PaO}_{2} \\
<75 \mathrm{mmHg}\end{array}$ & \multicolumn{2}{|c|}{1.163} & $<0.001$ \\
\hline $\begin{array}{c}<90 \mathrm{mmHg} \\
\text { Previous lung resection }\end{array}$ & 1 & & \\
\hline Right-side operation & 2 & 2.477 & $<0.001$ \\
\hline
\end{tabular}

Figure 2. ROC curve for the two groups

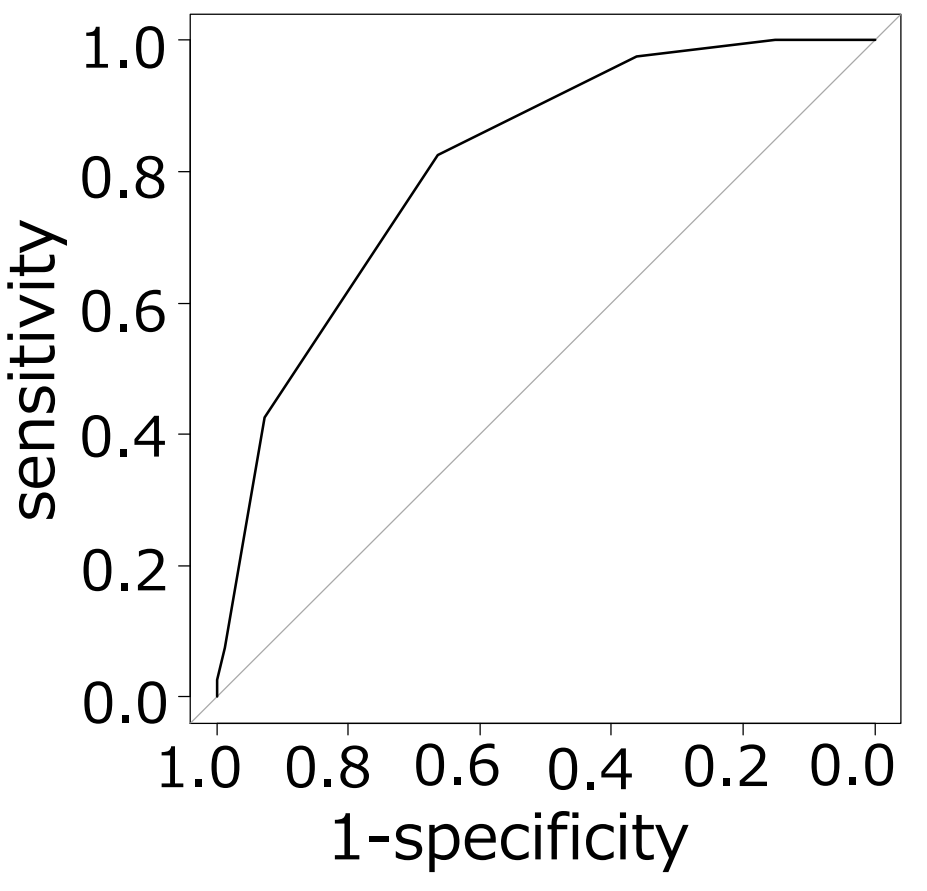

Derivation group C statistic 0.81

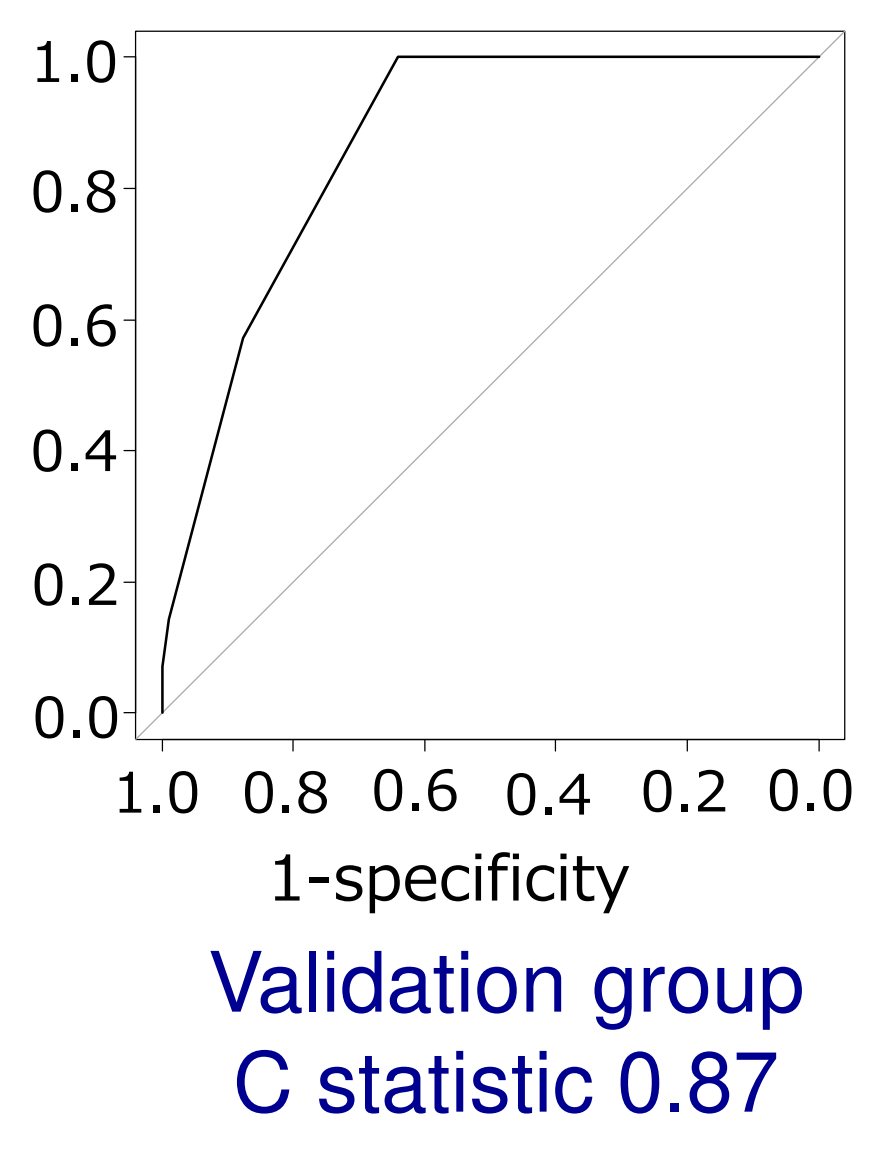

* Prediction score was developed using a regression coefficient-based scoring method.

\section{【Conclusion】}

The risk of hypoxemia during OLV was accurately predicted before surgery with this model constructed from preoperative low $\mathrm{PaO}_{2}$ value, history of lung resection, and the side of operation. 